\title{
RIGIDITY OF A CLASS OF SPECIAL LAGRANGIAN FIBRATIONS SINGULARITY *
}

\author{
JI-XIANG FU ${ }^{\dagger}$
}

1. Introduction. In [8], Strominger, Yau and Zaslow conjectured that the mirror pairs in mirror conjecture are pairs of dual special Lagrangian fibrations. Since then there has been a lot of research on special Lagrangian submanifolds or special Lagrangian fibrations. In this note, we will look at the possible singularities that can arise from a special Lagrangian fibrations. We will show that a fiber of a special Lagrangian fibration that has only isolated singularity of homogeneous type are essentially of the type given by Harvey-Lawson.

We let $\mathbb{C}^{3}$ be the complex 3-space endowed with the standard Kaehler metric with the associated Kaehler form $\omega_{0}$ and the $(3,0)$ form $\Omega_{0}=d z_{1} \wedge d z_{2} \wedge d z_{3}$. A submanifold $L \subset \mathbb{C}^{3}$ is called a special Lagrangian submanifold (in short SL-submanifold) if $\left.\omega_{0}\right|_{L}=$ 0 and $\left.\operatorname{Im}\left(\Omega_{0}\right)\right|_{L}=0$.

We let $S^{5} \subset \mathbb{C}^{3}$ be the unit sphere. For $p=\left(z_{1}, z_{2}, z_{3}\right) \in \mathbb{C}^{3}$ and $t \in \mathbb{R}$ we use $t p$ to denote the point $\left(t z_{1}, t z_{2}, t z_{3}\right) \in \mathbb{C}^{3}$. For any subset $\Sigma \subset S^{5}$ we define the cone supposed on $\Sigma$ to be

$$
C(\Sigma)=\left\{t p \mid t \in \mathbb{R}^{+}, p \in \Sigma\right\}
$$

We say $C(\Sigma)$ is an SL-cone if the smooth locus of $C(\Sigma)$ is dense in $C(\Sigma)$ and is an SL-submanifold of $\mathbb{C}^{3}$.

Now we introduce the notion of homogeneous SL-fibration of $\mathbb{C}^{3}$.

Definition 1. Let $F: \mathbb{C}^{3} \rightarrow \mathbb{R}^{3}$ be a smooth surjective map. We say $F$ is an SL-fibration if the components $f_{1}, f_{2}$ and $f_{3}$ of $F$ are real valued functions in $x_{1}, x_{2}, x_{3}, y_{1}, y_{2}, y_{3}$, where $z_{k}=x_{k}+i y_{k}$, so that all Poisson brackets $\left\{f_{i}, f_{j}\right\}=0$ and the real part $\operatorname{Re}\left\{\operatorname{det}_{\mathbb{C}}\left(\left(\partial f_{i} / \partial \overline{z_{j}}\right)\right)\right\}=0$. We say the fibration is homogeneous if all $f_{i}$ are homogeneous polynomials and we say the fiber $L_{0}=F^{-1}(0)$ is a regular cone if $L_{0}$ has only isolated singularity 0 and all $f_{i}$ are irreducible.

Recall that the smooth locus of any fibers of $F$ as in the Definition are automatically SL-submanifolds [3]. For convenience we denote the punctured cone $L_{0}-\{0\}$ by $L_{0}^{*}$. We first observe that in case $L_{0}$ is a regular cone, then $\operatorname{deg} f_{k} \geq 2$ for all $k$. Indeed, let $T_{0}$ be the linear combination of all tangent spaces of points in $L_{0}^{*}$, after translating to the origin 0 . We now show that $\operatorname{dim} T_{0}=6$. First, in case $\operatorname{dim} T_{0}=4$, then there are two points $p, q \in L_{0}^{*}$ so that $\operatorname{dim}\left(T_{p} L_{0}^{*} \cap T_{q} L_{0}^{*}\right)=2$. Because $T_{p} L_{0}^{*}$ and $T_{q} L_{0}^{*}$ are special Lagrangian subspaces in $\mathbb{C}^{3}$, we must have $T_{p} L_{0}^{*}=T_{q} L_{0}^{*}$, a contradiction. Now assume $\operatorname{dim} T_{0}=5$. Then there is an unit vector $J v \in T_{0}$. This implies that $J v$ is normal to $L_{0}^{*}$ everywhere and hence $v$ is a vector field of $L_{0}^{*}$. So we can write $L_{0}=t v \times \Gamma$, where $\Gamma$ is a cone of dimension 2 with singularity 0 . Thus $L_{0}$ has at least singularity $\mathbb{R}$, a contradition. Now from $\operatorname{dim} T_{0}=6$, we can easily obtain $\operatorname{deg} f_{k} \geq 2$ for all $k$. Moreover we can obtain that $\Sigma=L_{0} \cap S^{5}$ is full in $\mathbb{C}^{3}$.

The prototype of SL-fibration in $\mathbb{C}^{3}$ with homogeneous isolated singularity is the example of Harvey and Lawson [3].

*Received March 5, 2002; accepted for publication September 13, 2002. Project 10101004 supported by NSFC.

†Institute of Mathematics, Fudan University, China (jxfu1@online.sh.cn). 
EXAMPLE 2. Let $F=\left(f_{1}, f_{2}, f_{3}\right)$ be defined by

$$
f_{1}=\left|z_{1}\right|^{2}-\left|z_{2}\right|^{2}, f_{2}=\left|z_{1}\right|^{2}-\left|z_{3}\right|^{2} \quad \text { and } \quad f_{3}=\operatorname{Im}\left(z_{1} z_{2} z_{3}\right) \text {. }
$$

Then $F$ is a homogeneous $S L$-fibration of $\mathbb{C}^{3}$ and $L_{0}=F^{-1}(0)$ is a regular $S L$-cone.

So far this is the only known example of homogeneous SL-fibration of $\mathbb{C}^{3}$ whose central fiber is a regular cone. The fibration given by $\left(f_{1}, f_{2}, f_{3}\right): \mathbb{C}^{3} \rightarrow \mathbb{R}^{3}$ with

$$
f_{1}=x_{1} y_{2}-x_{2} y_{1}, \quad f_{2}=x_{1} y_{1}+x_{2} y_{2} \quad \text { and } f_{3}=y_{3} \text {. }
$$

is a homogeneous SL-fibration but its central fiber is not regular.

In this note, we will prove the following uniqueness result on homogeneous SLfibrations with singular central fibers.

THEOREM 3. Let $F=\left(f_{1}, f_{2}, f_{3}\right): \mathbb{C}^{3} \rightarrow \mathbb{R}^{3}$ be a homogeneous SL-fibration so that its central fiber $L_{0}$ is a regular cone. We let $n_{i}=\operatorname{deg} f_{i}$, so arranged that $n_{1} \leq n_{2} \leq n_{3}$. Then we must have $\left(n_{1}, n_{2}, n_{3}\right)=(2,2,3)$. Furthermore, there is a unitary matrix $S$ so that if we make the Darboux coordinates change

$$
\left(p_{1}, p_{2}, p_{3}, q_{1}, q_{2}, q_{3}\right)^{T}=S^{-1}\left(x_{1}, x_{2}, x_{3}, y_{1}, y_{2}, y_{3}\right)^{T}
$$

and let $w_{k}=p_{k}+i q_{k}$. Then $\left(f_{1}, f_{2}, f_{3}\right)$ is linearly equivalent to

$$
\tilde{f}_{1}=\left|w_{1}\right|^{2}-\left|w_{2}\right|^{2}, \quad \tilde{f}_{2}=\left|w_{1}\right|^{2}-\left|w_{3}\right|^{2} \quad \text { and } \quad \tilde{f}_{3}=\operatorname{Im}\left(w_{1} w_{2} w_{3}\right) .
$$

Acknowledgement. I like to take this opportunity to thank Jun $\mathrm{Li}$ for the invaluable advice and guidance. Without his insights and help, this work would not have been finished. I also thank Professor Gu Chaohao, Professor Hu Hesheng and Professor Xin Yuanlong for their guidance.

2. Harmonic 1-forms on SL cone. In this section, we collect a few facts concerning harmonic 1-forms on special Lagrangian cones in $\mathbb{C}^{3}$.

Let $L_{0}$ be an SL cone of $\mathbb{C}^{3}$ with isolated singularity 0 . The question we will address in section is whether there is a family of smooth proper SL-submanifolds $L_{s}$ of $\mathbb{C}^{3}$ such that $L_{s} \rightarrow L_{0}$ as $s \rightarrow 0$. In case such families exist, then on $L_{0}^{*}$ we have the associated normal vector field $W(x)$ and the associated 1-form $\theta(x)=W(x)\rfloor \omega_{0}$. In the following, we will call such 1-form the deformation 1-form associated to the family $L_{s}$. By a result of McLean, $\theta$ is a harmonic 1 -form on $L_{0}^{*}$. Further since $L_{s}$ are smooth $\theta$ is singular at 0 .

LEMMA 4. Let $L_{0}=C(\Sigma)$ be an $S L$-cone with isolated singularity 0 and let $t$ be the distance function $t(x)=\operatorname{dist}(x, 0)$ on $\mathbb{C}^{3}$. Then the space of harmonic 1 -forms on $L_{0}^{*}$ is spanned by

$$
t^{-1} \eta ; \quad d\left(t^{-1}\right) ; \quad d\left(t^{-\mu_{i}} \phi_{i}\right) \quad \text { and } \quad d\left(t^{\mu_{i}^{\prime}} \phi_{i}\right)
$$

where $\eta$ are harmonic 1 -forms on $\Sigma, \phi_{i}$ are eigenfunctions on $\Sigma$ with eigenvalues $\lambda_{i}>0$ with $\mu_{i}=\left(1+\sqrt{1+4 \lambda_{i}}\right) / 2$ and $\mu_{i}^{\prime}=\left(-1+\sqrt{1+4 \lambda_{i}}\right) / 2$.

Proof. Let $\tau=\frac{\partial}{\partial t}$ be the unit tangent vector field on $L_{0}^{*}$. Let $m_{0} \in \Sigma$ be any point. We pick an orthonormal vector fields $\left(e_{1}, e_{2}\right)$ on $\Sigma$ near $m_{0}$ that is covariantly constant at $m_{0}$ with respect to the connection on $\Sigma$. We then extend the vector fields $e_{k}$, considered as vector fields of $\Sigma \subset S^{5} \subset \mathbb{C}^{3}$, to the cone $L_{0}^{*}$ by parallel translation 
along rays in the cone. Combined with $\tau$, we obtain an orthonormal frame of $L_{0}^{*}$ near $\mathbb{R} m_{0}$. Now let $l: \mathbb{R}^{+} \times \Sigma \rightarrow C(\Sigma) \subset \mathbb{C}^{3}$ be defined via $l(t, m)=t m$. We give $\mathbb{R}^{+} \times \Sigma$ the metric $\widetilde{g}=d t^{2}+t^{2} d_{\Sigma}^{2}$, where $d_{\Sigma}^{2}$ is the metric on $\Sigma$. We let $\tau: \mathbb{R}^{+} \times \Sigma \rightarrow \Sigma$ be the project. Then $\left(\tau, E_{1}(r, m), E_{2}(t, m)\right)$ forms an orthonormal frame on $\mathbb{R}^{+} \times \Sigma$ with $\tau=\frac{\partial}{\partial t}, E_{1}(t, m)=\frac{1}{t} \tau^{*} e_{1}(m)$ and $E_{2}(t, m)=\frac{1}{t} \tau^{*} e_{2}(m)$. Its dual frame is given by $d t, \omega_{1}(t, m)=t \tau^{*} \omega_{1}(m)$ and $\omega_{2}(t, m)=t \tau^{*} \omega_{2}(m)$. Clearly, we have

$$
l_{*} E_{i}(t, m)=e_{i}(t m)=e_{i}(m) \text { and } l^{*} \omega_{i}(t m)=l^{*} \omega_{i}(m)=\omega_{i}(t, m)
$$

On $L_{0}^{*}$ we have the structure equation

$$
d \omega_{i}(t m)=-\omega_{i j}(t m) \wedge \omega_{j}(t m)-t^{-1} \omega_{i}(t m) \wedge d t
$$

Our convention is that we use $(t, m)$ to denote the point in $\mathbb{R}^{+} \times \Sigma$ while we use $t m$ to denote the corresponding point in $C(\Sigma)$. Note $l(t, m)=t m$. Over $\mathbb{R}^{+} \times \Sigma$ we have the structure equation

$$
d \omega_{i}(t, m)=d\left(l^{*} \omega_{i}(t m)\right)=-l^{*}\left(\omega_{i j}(t m)\right) \wedge \omega_{j}(t, m)-t^{-1} \omega_{i}(t, m) \wedge d t
$$

Let harmonic 1-form

$$
\theta=f(t m) d t+\omega(t m)=f(t m) d t+\sum \alpha_{i}(t m) \omega_{i}(t m)
$$

then

$$
l^{*} \theta=f(t, m) d t+\sum \alpha_{i}(t, m) \omega_{i}(t, m) .
$$

From $d l^{*} \theta=0$, we obtain

$$
t \nabla_{E_{i}(t, m)} f(t, m)-\frac{\partial}{\partial t}\left(t \alpha_{i}(t, m)\right)=0, \text { for } i=1,2
$$

Using the above equation, a straight forward computation shows that

$$
d\left(\int_{1}^{t} f(r, m) d r\right)=l^{*} \theta-\tau^{*} \eta(m)
$$

where $\eta(m)=\sum_{i=1}^{2} \alpha_{i}(1, m) \omega_{i}(m)$, a 1 -form on $\Sigma$. Because $l^{*} \theta$ is harmonic on $\mathbb{R}^{+} \times \Sigma, \tau^{*} \eta$ is closed on $\mathbb{R}^{+} \times \Sigma$ and hence $\eta$ must be closed on $\Sigma$. Now let $\eta_{h}$ be the harmonic part of $\eta$, namely, $\eta=\eta_{h}+d k$. Then

$$
l^{*} \theta=d\left(\int_{1}^{t} f(r, \cdot) d r+\tau^{*} k\right)+\tau^{*} \eta_{h}=d F+\tau^{*} \eta_{h}
$$

where $F=\int_{1}^{t} f(r, \cdot) d r+\tau^{*} k$. Clearly, $\tau^{*} \eta_{h}$ is harmonic on $\mathbb{R}^{*} \times \Sigma$. Since $l^{*} \theta$ is harmonic, $d F$ must be harmonic. Hence by [7, page 98$]$

$$
\Delta_{\Sigma}(F(t, m))+2 t \frac{\partial}{\partial t} F(t, m)+t^{2} \frac{\partial^{2}}{\partial t^{2}} F(t, m)=0 .
$$

Now let $\phi_{i}$ be the eigenfunctions on $\Sigma$ with eigenvalues $\lambda_{i}$. Then

$$
F(t, m)=\sum_{i=0}^{\infty} f_{i}(t) \phi_{i}(m)
$$


for some functions $f_{i}(t)$. From this we obtain

$$
\sum_{i=0}^{\infty}\left(-\lambda_{i} f_{i}(t)+2 t f_{i}^{\prime}(t)+t^{2} f_{i}^{\prime \prime}(t)\right) \phi_{i}(m)=0 .
$$

Therefore $f_{i}$ satisfies the equation

$$
-\lambda_{i} f_{i}(t)+2 t f_{i}^{\prime}(t)+t^{2} f_{i}^{\prime \prime}(t)=0
$$

whose general solutions are $f_{i}=C_{i 1} t^{\mu_{i}^{\prime}}+C_{i 2} t^{-\mu_{i}}$ with $\mu_{i}^{\prime}=\left(-1+\sqrt{1+4 \lambda_{i}}\right) / 2$ and $\mu_{i}=\left(1+\sqrt{1+4 \lambda_{i}}\right) / 2$.

When $i=0$ then $\mu_{0}=1$ and $\phi_{0}$ is a constant. In this case $d\left(t^{-1} \phi_{0}\right)$ reduces to the 1 -form $d\left(\frac{1}{t}\right)$.

We now compute the deformation 1-forms of two examples of smoothing of SLcones. We begin with the deformation 1-forms of Harvey-Lawson's example. Let $\theta_{k}$ be the deformation 1 -form on associated to the family $L_{s}^{[k]}$ defined by $f_{k}=s$ and $f_{j}=0$ for $j \neq k$. Here $\left(f_{1}, f_{2}, f_{3}\right)$ is the defining equation in Example 2. Then by a direct computation we have $\theta_{1}=\frac{3}{2}\left(d \alpha_{1}-2 d \alpha_{2}+d \alpha_{3}\right), \theta_{2}=\frac{3}{2}\left(d \alpha_{1}+d \alpha_{2}-2 d \alpha_{3}\right)$ and $\theta_{3}=\frac{1}{3} d\left(\frac{1}{t}\right)$. Here $\alpha_{k}$ is the function on $\Sigma$ defined by $x_{k}=r \cos \alpha_{k}, y_{k}=r \sin \alpha_{k}$. Note that $\left.\theta_{1}\right|_{\Sigma}$ and $\left.\theta_{2}\right|_{\Sigma}$ are harmonic 1 -forms on the 2-torus $\Sigma$.

Now we consider the case of a homogeneous SL-fibration $F=\left(f_{1}, f_{2}, f_{3}\right): \mathbb{C}^{3} \rightarrow$ $\mathbb{R}^{3}$ whose central fiber is a regular cone, as defined in Definition 1 . We let $L_{s}^{[i]}$ be the family $\left\{f_{i}=s, f_{j}=0\right.$ for $\left.j \neq i\right\}$ and let $W_{i}$ be the deformation vector field associated to the family $L_{s}^{[i]}$.

Lemma 5. Let $W_{1}=\sum_{i} c_{1 i} \partial_{x_{i}}+c_{1 i+3} \partial_{y_{i}}$ be the normal deformation vector field of the family $L_{s}^{[1]}$ at $L_{0}$. Then $c_{1 i}$ can be written as $c_{1 i}=h_{i} / g_{i}$, where $h_{i}$ and $g_{i}$ are homogeneous polynomials with $\operatorname{deg} h_{i}-\operatorname{deg} g_{i}=1-n_{1}$ for $1=1, \cdots, 6$.

Proof. Normal deformation vector field $W_{1}$ satisfies the following equation:

$$
A \cdot\left(c_{11}, c_{12}, \cdots, c_{16}\right)^{T}=(1,0, \cdots, 0)^{T}
$$

where

$$
A=\left[\begin{array}{cc}
\frac{\partial f_{i}}{\partial x_{j}} & \frac{\partial f_{i}}{\partial y_{j}} \\
-\frac{\partial f_{i}}{\partial y_{j}} & \frac{\partial f_{i}}{\partial x_{j}}
\end{array}\right]_{1 \leq i, j \leq 3}
$$

So,

$$
\left(c_{11}, c_{12}, \cdots, c_{16}\right)^{T}=A^{-1}(1,0, \cdots, 0)^{T}=(\operatorname{det} A)^{-1}\left(A_{11}, A_{12}, \cdots, A_{16}\right)^{T}
$$

Because $\operatorname{deg}(\operatorname{det} A)=2\left(n_{1}+n_{2}+n_{3}\right)-6$ and $\operatorname{deg}\left(A_{1 i}\right)=2\left(n_{2}+n_{3}\right)-5$, we can write $c_{1 i}=h_{i} / g_{i}$ with $h_{i}$ and $g_{i}$ are homogeneous and $\operatorname{deg} h_{i}-\operatorname{deg} g_{i}=1-n_{1}$.

In this case we say the deformation 1 -form $\left.\theta_{1}=W_{1}\right\rfloor \omega_{0}$ has order $n_{1}-1$.

The previous examples show that deformation 1-forms associated to the families $L_{s}$ are spanned by 1 -forms $t^{-1} \eta$ or $d\left(t^{-1}\right)$. In following, we will study a class of SL-submanifolds that has similar property.

DEFINITION 6. Let $L$ be a smooth $S L$-submanifold in $\mathbb{C}^{3}$ and let $L_{0}$ be an $S L$-cone in $\mathbb{C}^{3}$ with isolated singularity 0 . We say $L$ is asymptotically conical (in short $A C$ ) to 
$L_{0}$ and call $L_{0}$ the asymptotic cone of $L$ if $L$ is asymptotic to $L_{0}$ to order $O\left(t^{-1}\right)$ as $t \rightarrow \infty$.

Clearly, when $L$ is AC to $L_{0}$ than $s L$ is also AC to $L_{0}$ for $s \in \mathbb{R}^{+}$and $s L \rightarrow L_{0}$ as $s \rightarrow 0$. To obtain a deformation 1 -form on $L_{0}^{*}$ we need certain convergence condition on $s L$.

Definition 7. Let $L$ be a smooth $S L$-submanifold in $\mathbb{C}^{3}$ that is $A C$ to $L_{0}$. We assume $L_{0}=C(\Sigma)$ for a smooth $\Sigma \subset S^{5}$. We say $L$ is strongly $A C$ to $L_{0}$ if there is a constant $q>0$ and a smooth maps $\Phi(\cdot, s): \Sigma \times[0,1] \rightarrow S^{5}$ such that

(1) $\Phi(\Sigma, s)=\left(s^{1 / 1+q} L\right) \cap S^{5}$ and $\Phi(\cdot, s)$ is a diffeomorphism from $\Sigma$ to its image;

(2) The family of maps $H_{s}(p, t)=t \Phi\left(p, s / t^{1+q}\right)$ from $\Sigma \times[1, \infty) \rightarrow \mathbb{C}^{3} C^{1}$-converges to the standard map $\Sigma \times[1, \infty) \rightarrow L_{0}-B_{1}$ when $s \rightarrow 0$, where $B_{1}$ is the unit ball in $\mathbb{C}^{3}$;

(3) The vector field $\mathbf{v}(p)=\left.\frac{d}{d s}\right|_{s=0} \Phi(p, s)$ is a non-trivial vector field on each connected component of $\Sigma \subset S^{5}$.

The notion of SL-submanifolds AC to a cone was introduced in [5].

EXAMPLE. [3] Let

$$
L_{s}=\left\{(x, y) \in \mathbb{C}^{3}|| x|y=| y \mid x \text { and } \operatorname{Im}(|x|+i|y|)^{3}=s\right\}
$$

Then $L_{s}$ is a SL-submanifold strongly $\mathrm{AC}$ to a cone $L_{0}$ that is the union of two linear subspaces singular at 0 . The associated $q$ is 2 in this case.

EXAMPLE. Let $F=\left(f_{1}, f_{2}, f_{3}\right): \mathbb{C}^{3} \rightarrow \mathbb{R}^{3}$ be a homogeneous SL-fibration whose central fiber is a regular cone. We let $n_{i}=\operatorname{deg} f_{i}$ with $f_{i}$ so arranged that $n_{1} \leq n_{2} \leq$ $n_{3}$. By Sard theorem, there is a point $\xi \neq 0 \in \mathbb{R}^{3}$ such that $L=F^{-1}(\xi)$ is a smooth complete SL-submanifold. Then $L$ is strongly AC to the cone $L_{0}=F^{-1}(0)$. The associated $q$ is $1 / n_{i}$ where $i$ is the smallest index so that $\xi_{i} \neq 0$.

Let $L$ be an SL-submanifold which is strongly AC to an SL-cone $L_{0}$ with the associated constant $q$. It follows from the definition that the deformation 1-form on $L_{0}^{*}$ associated to the family $L_{s}=s^{1 / 1+q} L$ is a smooth non-trivial 1-form on $L_{0}^{*}$.

LEMMA 8. Let $L, L_{0}$ and $q$ be as before and let $\theta$ be the deformation 1-form on $L_{0}^{*}$ associated to the family $L_{s}$. Then $q$ can only take values 1,2 or $\mu_{i}+1$, where $\mu_{i}=\left(1+\sqrt{1+4 \lambda_{i}}\right) / 2$. Further, when $q=1$ (resp. $q=2$; resp. $q=\mu_{i}+1$ ) the form $\theta=t^{-1} \eta\left(\right.$ resp. $\theta=d t^{-1}$; resp. $\left.\theta=d\left(t^{-\mu_{i}} \phi_{i}\right)\right)$, where $\eta$ and $\phi_{i}$ are as in Lemma 4 .

Proof. Let $\mathbf{v}(p)$ be the vector field on $L_{0}^{*}$ that is the limit of $\frac{d}{d s} L_{s}$ when $s \rightarrow 0$. Then by definition, $\mathbf{v}(p)$ is non-trivial on each component of $L_{0}^{*}$. By the definition of $H_{s}$, it is direct to check that $\mathbf{v}(t p)=t^{-q} \mathbf{v}(p)$. Hence the deformation 1-form $\theta$ on $L_{0}$ is also homogeneous, which must be of the forms $\frac{1}{t} \eta, d\left(\frac{1}{t}\right)$, or $d\left(t^{-\mu_{i}} \phi_{i}\right)$.

3. Harmonic 1-forms on SL-submanifolds. Let $L$ be an SL-submanifold that is strongly $\mathrm{AC}$ to an SL-cone $L_{0}$. We first give $L$ a new metric that is quasi-isometric to its induced metric $g$.

LEMMA 9. Let the notation be as before. Then there is a metric $\bar{g}$ on $L$ that is quasi-isomorphic to $(L, g)$ so that $\bar{g}$ is isomorphic to a cone metric away from a compact subset of $L$.

Proof. The proof is standard. It follows from the $C^{1}$ convergence of $H_{t}$ in Definition 7. 
Let $M$ be a complete non-compact Riemannian manifold. We recall several groups associated to $M$. Here we use $\|\theta\|_{2}$ to mean the $L_{2}$ norm $\int_{M}|\theta|^{2}$ and use $L^{2}$ to denote the space of $L^{2}$ finite functions or forms. We define

$$
\mathcal{H}_{D}(M)=\left\{f \mid \Delta f=0, d f \in L^{2}\right\} \quad \text { and } \quad \mathcal{H}_{D}^{\infty}(M)=\left\{f \in \mathcal{H}_{D}(M) \mid f \in L^{\infty}\right\}
$$

and

$$
\mathcal{H}^{1}(M)=\left\{\theta \mid d \theta=\delta \theta=0, \theta \in L^{2}\right\} \quad \text { and } \quad \mathcal{H}_{0}^{1}(M)=\left\{d f \mid \Delta f=0, d f \in L^{2}\right\} .
$$

Here $f$ are functions and $\theta$ are one forms on $M$. We also define

$$
H_{(2)}^{1}(M)=\left\{\theta \mid d \theta=0, \theta \in L^{2}\right\} /\left\{d f \mid f \in L^{2}, d f \in L^{2}\right\} .
$$

First, recall that we have the Hodge decomposition $\mathcal{H}^{1}(M) \cong H_{(2)}^{1}(M)$ [4]. In the following, we will apply a theorem P.Li and L-H.Tam [6, Thm 4.2] to prove the following fact.

LEMma 10. Let $L$ be an $S L$-submanifold strongly $A C$ to an $S L$-cone $L_{0}$ endowed with the induced metric $g$ and let $\bar{L}$ be $L$ endowed with the metric $\bar{g}$ given in Lemma 9. Then we have

$$
\operatorname{dim} \mathcal{H}_{D}^{\infty}(\bar{L})=\operatorname{dim} \mathcal{H}_{D}^{\infty}(L)=\#(\text { ends of } L)
$$

Proof. Let $K(R)$ be $B(R) \cap L$. By the construction, for large enough $R$ the compliment $L-K(R)$ with metric $\bar{g}$ is a union of cones. Our strategy is to apply [6, Theorem 4.2] to $(\bar{L}, \bar{g})$.

We now check that this theorem can be applied in our situation. First, $\bar{L}$ is large because $L$ is strongly $\mathrm{AC}$ to the cone $L_{0}$. Now let $E$ be an end of $L-K(R)$. To proceed, we need to check that there is a constant $C$ so that the Ricci curvature of $(E, \bar{g})$ satisfies

$$
\operatorname{Ric}(x) \geq-\frac{2 C}{(1+r(x))^{2}}
$$

where $r(x)=\operatorname{dist}(p, x)$ for a $p$ in $E$. Because $C(\Sigma)$ is minimal, we have

$$
R_{i i}=\sum_{\alpha, j}\left(h_{j j}^{\alpha} h_{i i}^{\alpha}-\left(h_{i j}^{\alpha}\right)^{2}\right)=-\sum_{\alpha, j}\left(h_{i j}^{\alpha}\right)^{2}
$$

where $h_{i j}^{\alpha}=h_{i j}^{\alpha}(t, m)$ is the second fundamental form of $C(\Sigma)$. Now let $h_{i j}^{\alpha}(m)$ be the second fundamental form of $\Sigma$ in $S^{5}$. Because $h_{i j}^{\alpha}(t, m)=\frac{1}{t} h_{i j}^{\alpha}(m)$ (on $C(\Sigma)$ ), we have

$$
R_{i i}(m, t)=-t^{-2} \sum_{\alpha, j}\left(h_{i j}^{\alpha}(m)\right)^{2} \geq t^{-2} C_{1}
$$

for $C_{1}=\sup _{i, m} \sum_{j, \alpha}\left(h_{i j}^{\alpha}(m)\right)^{2}$. Because $t^{2} \sim r^{2}(x)$, there is a constant $C_{2}$ so that $R_{i i}(x) \geq-\frac{C_{2}}{r(x)^{2}}$ on $E$. This shows that there is a constant $C$ so that (3.1) holds.

Finally, we need to check that $E$ satisfies the condition (VC) in [6, p.282]. Namely, there is a constant $\zeta>0$ such that for all $r$ and all $x \in \partial B_{p}(r) \cap E$, we have $V_{p, E}(r)<\zeta V_{x, E}\left(\frac{r}{2}\right)$. First, it is clear that it suffices to check this condition for $r \geq R^{\prime}$ 
for some constant $R^{\prime}$. We choose an $R^{\prime}$ so that $\partial B_{p}\left(R^{\prime}\right) \subset E$ and that for any $x \in \partial B_{p}\left(R^{\prime}\right), B_{x}\left(R^{\prime} / 2\right) \cap E \subset \bar{L}-K(R)$. Then when $r \geq R^{\prime}$, we have

$$
V_{x, E}\left(\frac{r}{2}\right)=V_{\frac{R^{\prime}}{r} x, E}\left(R^{\prime} / 2\right) \cdot \frac{r^{3}}{R^{\prime 3}}
$$

Let $\zeta_{1}=\min _{x \in \partial B_{p}\left(R^{\prime}\right) \cap E} V_{x, E}\left(\frac{R^{\prime}}{2}\right)$. Then $V_{x, E}(r / 2)>\zeta_{1} R^{\prime-3} r^{3}$. Since $V_{p, E}(r) \approx \operatorname{Area}(\Sigma)\left(r^{3}-(2 R)^{3}\right)$, therefore there is a constant $\zeta$ so that $V_{p, E}(r)<\zeta V_{x, E}(r / 2)$.

This shows that we can apply [6, Theorem 4.2] to $\bar{L}$ to conclude that the dimension of $\mathcal{H}_{D}^{\infty}(\bar{L})$ is equal to the number of ends of $\bar{L}$. Since $L$ is quasi-isometric to $\bar{L}$, $\operatorname{dim} \mathcal{H}_{D}^{\infty}(L)=\operatorname{dim} \mathcal{H}_{D}^{\infty}(\bar{L})$ [2]. Therefore $\operatorname{dim} \mathcal{H}_{D}^{\infty}(L)$ is equal to the number of ends on $L$. $\square$

LEMMA 11. Let $L$ be a SL submanifold which is strongly $A C$ to $S L$ cone $L_{0}$, then $\operatorname{dim} \mathcal{H}_{0}^{1}(\bar{L})=\#\{$ ends of $L\}-1$.

Proof. From Lemma 4 , we obtain $\mathcal{H}_{D}(\bar{L})=\mathcal{H}_{D}^{\infty}(\bar{L})$. Then the Lemma follows from $\operatorname{dim} \mathcal{H}_{0}^{1}(\bar{L})=\operatorname{dim} \mathcal{H}_{D}(\bar{L})-1$ and Lemma 10 .

Let

$$
H_{c}^{1}(L)=\{\theta \mid d \theta=0, \theta \text { has compact support }\} /\{d f \mid f \text { has compact support }\}
$$

and let $H^{1}(L)$ be the first de Rham cohomology group. Consider the natural map $i_{1}^{*}: H_{c}^{1}(L) \rightarrow H^{1}(L)$. Then

$\operatorname{Ker}\left(i_{1}^{*}\right)=\{d g \mid d g$ has compact support $\} /\{d f \mid f$ has compact support $\}$

We continue to assume that $L$ is an SL-submanifold strongly AC to an SL-cone $L_{0}$.

LEMMA 12. $\operatorname{Ker}\left(i_{1}^{*}\right)=\#($ ends of $L)-1$.

Proof. Let $[d g] \neq 0 \in \operatorname{Ker}\left(i_{1}^{*}\right)$. Because $L$ is strongly AC to $L_{0}$, without loss of generality, we can assume that the compact subset $K \subset L$ of $d g$ is so large that $L-K$ is diffeomorphic to the disjoint union of $\Sigma_{i} \times(0, \infty)$. Hence when restricted to the ends $L-K g$ is locally constant but not constant. Now we see that $\operatorname{Ker}\left(i_{1}^{*}\right)=$ \#(ends of $L)-1$.

Now we consider $\operatorname{Im}\left(i_{1}^{*}\right)$. From [4, Page 9], any compactly supported cohomology class on a complete Riemannian manifold that defines a non-trivial de Rham cohomology class is automatically represented by an $L^{2}$-harmonic form. This defines a natural Hodge projective

$$
\pi: \operatorname{Im}\left(i_{1}^{*}\right) \longrightarrow \mathcal{H}^{1}(\bar{L})
$$

Define

$$
[\pi]: \operatorname{Im}\left(i_{1}^{*}\right) \longrightarrow \mathcal{H}^{1}(\bar{L}) / \mathcal{H}_{0}^{1}(\bar{L})
$$

Lemma 13. Let $L$ be an $S L$-submanifold strongly $A C$ to an $S L$-cone $L_{0}$, then

$$
\operatorname{Im}\left(i_{1}^{*}\right) \cong \mathcal{H}^{1}(\bar{L}) / \mathcal{H}_{0}^{1}(\bar{L})
$$


and the isomorphism is induced by above Hodge projection.

Proof. Clearly, $[\pi]$ is injective. And we must prove $[\pi]$ is surjective.

Let $[\omega] \in \mathcal{H}^{1}(\bar{L}) / \mathcal{H}_{0}^{1}(\bar{L})$. Because $L$ is strongly $\mathrm{AC}$ to $L_{0}$, we can pick a sufficiently large compact subset $K\left(2 r_{0}\right) \subset L$ so that $L-K\left(2 r_{0}\right)$ is diffeomorphic to the disjoint union of $\Sigma_{i} \times(0, \infty)$. We let $\Lambda_{i}$ be the $i$ th component of $\bar{L}-K\left(2 r_{0}\right)$. Then from lemma 4 , we can write

$$
\left.\omega\right|_{\Lambda_{i}}=C_{i} \cdot \frac{1}{t} \eta_{i}+d f_{i}
$$

where $C_{i}$ is some constant and $\eta_{i}$ is some harmonic 1 -form on $\Sigma_{i}, f_{i}$ is a harmonic function on $\Lambda_{i}$. If $C_{i} \neq 0$ for some $i$, then

$$
\int_{\Lambda_{i}}\left|C_{i} \cdot \frac{1}{t} \eta_{i}\right|^{2}=+\infty
$$

and

$$
\int_{\Lambda_{i}}<C_{i} \cdot \frac{1}{t} \eta_{i}, d f_{i}>=0
$$

Thus $\omega \notin \mathcal{H}^{1}(\bar{L})$. So we can write $\left.\omega\right|_{\bar{L}-K\left(2 r_{0}\right)}=d f$, for some harmonic function $f$ on $\bar{L}-K\left(2 r_{0}\right)$. Then we can write $\omega=(\omega-d(\rho f))+d(\rho f)$, where function $\rho$ with takes values between 0 and 1 and such that

$$
\rho(x)=1 \text {, for } x \in L-K\left(2 r_{0}\right) \text { and } \rho(x)=0 \text {, for } x \in K\left(r_{0}\right) .
$$

Thus, $\theta=\omega-d(\rho f)$ has compact support $K\left(2 r_{0}\right)$. But from the Hodge projection $\pi$, we can write $\theta=\pi \theta+d \varphi$ for some function $\varphi$ with $\int|d \varphi|^{2}<+\infty$. So we have $\omega=\pi \theta+d \varphi+d(\rho f)=\pi \theta+d(\varphi+\rho f)$ with $\int|d(\varphi+\rho f)|^{2}<+\infty$ and thus $d(\varphi+\rho f) \in \mathcal{H}_{0}^{1}(L)$. So $[\pi] \theta=[\omega]$.

TheOREM 14. Let $L$ be an $S L$ submanifold which is strongly $A C$ to an $S L$ cone, then $\operatorname{dim} \mathcal{H}^{1}(L)=\operatorname{dim}_{c}^{1}(L)$.

Proof. From lemma 11, 12 and 13, we have $\operatorname{dim} \mathcal{H}^{1}(\bar{L})=\operatorname{dim} H_{c}^{1}(L)$. Combined with $\mathcal{H}^{1}(L) \cong \mathcal{H}^{1}(\bar{L})$, we prove the Lemma.

\section{Proof of the main result.}

LEMma 15. Let $F=\left(f_{1}, f_{2}, f_{3}\right): \mathbb{C}^{3} \rightarrow \mathbb{R}^{3}$ be a homogeneous $S L$ fibration so that its central fiber $L_{0}$ is a regular cone. Then every connected component $L$ of regular fiber is diffeomorphic to $\mathbb{R} \times T^{2}, \mathbb{R}^{2} \times S^{1}$ or $\mathbb{R}^{3}$.

Proof. Let

$$
g_{i}=\frac{f_{i}^{2}}{1+f_{i}^{2}}, \text { for } i=1,2,3
$$

then

$$
\begin{array}{r}
\left\{g_{i}, g_{j}\right\}=<J \operatorname{grad} g_{i}, \operatorname{grad} g_{j}> \\
=\frac{2 f_{i} f_{j}}{\left(1+f_{i}^{2}\right)^{2}\left(1+f_{j}^{2}\right)^{2}}<J \operatorname{grad} f_{i}, \operatorname{grad} f_{j}>=0 .
\end{array}
$$


So $G=\left(g_{1}, g_{2}, g_{3}\right): \mathbb{C}^{3} \rightarrow \mathbb{R}^{3}$ defines a 3 -degree of freedom Liouville integrable Hamiltonian system. Because $f_{i}$ are homogeneous polynomials, Hamiltonian vector fields $X_{g_{i}}$ are bounded on $\mathbb{C}^{3}$. So from a theorem in [10, Cor 2, p.17] solutions of Cauchy problem: $\frac{d z}{d t}=X_{g_{i}}, z\left(t_{0}\right)=z_{0}$ are complete, i.e., $X_{g_{i}}$ are complete. Thus by the generalized Liouville theorem, every connected component of regular fiber $G^{-1}\left(v_{1}, v_{2}, v_{3}\right)$ is differential homeomorphic to $\mathbb{R} \times T^{2}, \mathbb{R}^{2} \times S^{1}, \mathbb{R}^{3}$ or $T^{3}$. Now every connected component $L$ of regular fiber $F^{-1}\left(s_{1}, s_{2}, s_{3}\right)$ is a connected component of regular fiber $G^{-1}\left(v_{1}, v_{2}, v_{3}\right)$, where $v_{i}=\frac{s_{i}^{2}}{1+s_{i}^{2}}$. So $L$ is differential homeomorphic to $\mathbb{R} \times T^{2}, \mathbb{R}^{2} \times S^{1}, \mathbb{R}^{3}$ or $T^{3}$. But from Example in section 2 , we know that $L$ is strongly AC to SL cone and is not compact, so $L$ is not diffeomorphic to $T^{3}$.

Now we can prove the following

Proposition 16. Let $F=\left(f_{1}, f_{2}, f_{3}\right): \mathbb{C}^{3} \rightarrow \mathbb{R}^{3}$ be a homogeneous SL-fibration so that its central fiber $L_{0}$ is a regular cone. We let $n_{i}=\operatorname{deg} f_{i}$, so arranged that $n_{1} \leq n_{2} \leq n_{3}$. Then we must have $\left(n_{1}, n_{2}, n_{3}\right)=(2,2,3)$.

Proof. By observation in section 1 , we have $\operatorname{deg} f_{k} \geq 2$ for all $k$. Let $\theta_{i}$ denote the deformation 1-form associated to the family $L_{s_{i}}=\left\{f_{i}=s_{i} ; f_{j}=0\right.$ for $\left.j \neq i\right\}$. Because we assume that $f_{i}$ are irreducible, $\theta_{1}, \theta_{2}$ and $\theta_{3}$ are linearly independent at any point $p \in L_{0}^{*}$. Note that $d t$ is 1 -form in $L_{0}^{*}$. Now we prove the proposition by studying case by case:

Case 1: $\operatorname{deg} F=\left(2,2, n_{3}\right)$ with $n_{3} \neq 3$.

In this case, $\theta_{1}$ and $\theta_{2}$ have order 1 . By Lemma 4, we can let $\theta_{1}=\frac{1}{t} \eta_{1}$ and $\theta_{2}=\frac{1}{t} \eta_{2}$, where $\eta_{1}$ and $\eta_{2}$ are harmonic 1 -forms on $\Sigma=L_{0} \cap S^{5}$. Now if $n_{3}=2$, we can also let $\theta_{3}=\frac{1}{t} \eta_{3}$, where $\eta_{3}$ is the harmonic form on $\Sigma$. Thus $\left\langle\theta_{i}, d t\right\rangle=0$ for $i=1,2,3$ and $\theta_{1}, \theta_{2}$ and $\theta_{3}$ are linearly dependent on $L_{0}^{*}$. This is impossible. If $n_{3} \geq 4$, by Lemma 4 we can let $\theta_{3}=d\left(t^{-\mu_{i}} \phi_{i}\right)$, where $\phi_{i}$ is an eigenfunction of $\Sigma$ which is not constant. Since $\Sigma$ is compact, we know that there is a point $p$ of $\Sigma$ such that $\phi_{i}(p)=0$. So $\theta_{3}(p)$ doesn't contain $d t$ as component at point $p$ and thus $\theta_{1}, \theta_{2}$ and $\theta_{3}$ are linearly dependent at point $p$.

Case 2: $\operatorname{deg} F=\left(2,3, n_{3}\right)$.

In this case. the deformation 1 -form $\theta_{2}$ of $L_{s_{2}}$ has order 2. Hence $\theta_{2}=C_{2} d\left(\frac{1}{t}\right)$ and therefore $n_{3}$ can not be 3 . The 1 -form associated to the family $L_{s_{3}}$ can be written $\theta_{3}=d\left(t^{-\mu_{i}} \phi_{i}\right)$, where $\phi_{i}$ is a non-constant eigenfunction on $\Sigma$. Because $\Sigma$ is compact, then there is a point $m \in \Sigma$ such that $\phi_{i}$ attains maximum at point $m$. So $\theta_{3}(m)=\phi_{i}(m) d t^{-\mu_{i}}$, and $\theta_{2}(m)$ and $\theta_{3}(m)$ is linearly dependent at point $m$. This is a contradiction.

Case 3: $\operatorname{deg} F=\left(n_{1}, n_{2}, n_{3}\right)$ with $n_{3} \geq n_{2} \geq 4$.

If there exists such $F$, then there is a point $s_{0}=\left(s_{10}, s_{20}, s_{30}\right) \in \mathbb{R}^{3}$, such that $L_{s_{0}}=F^{-1}\left(s_{10}, s_{20}, s_{30}\right)$ is a smooth SL submanifold. We have proven that $L_{s_{0}}$ is strongly $\mathrm{AC}$ to an SL cone $L_{0}$ in the example before Lemma 8. Let $L$ be a connected component of $L_{s_{0}}$. By Theorem 14, we have $\operatorname{dim} \mathcal{H}^{1}(L)=\operatorname{dim} H_{c}^{1}(L)$. By Poincare Lemma and Lemma 15, we have $\operatorname{dim} H_{c}^{1}(L) \leq 1$. Thus $\operatorname{dim} \mathcal{H}^{1}(L) \leq 1$.

Because $L$ is smooth, we can get three deformation 1 -forms $\theta_{1}, \theta_{2}$ and $\theta_{3}$. Certainly these forms are harmonic on $L$. But we assume that $n_{3} \geq n_{2} \geq 4$, so $\theta_{2}$ and $\theta_{3}$ has order at least 3 . Now by the homogeneous, we can get $\theta_{2}, \theta_{3} \in \mathcal{H}^{1}(L)$, which contradicts to $\operatorname{dim} \mathcal{H}^{1}(L) \leq 1$. 
In order to discuss the case of $\left(n_{1}, n_{2}, n_{3}\right)=(2,2,3)$, we need the following Lemma. In the following we denote by $\operatorname{diag}\left(a_{1}, a_{2}, \cdots, a_{n}\right)$ the $n \times n$ diagonal matrix with diagonal entries $a_{1}, a_{2}, \cdots, a_{n}$.

LEMMA 17. Let $\widetilde{A}=\operatorname{diag}(1,-k, 0,1,-k, 0)$ with $k>0$ and let matrix $\widetilde{B}$ be symmetric such that (1) $\widetilde{A} E \widetilde{B}=\widetilde{B} E \widetilde{A}$, where matrix $E$ has the form

$$
E=\left(\begin{array}{cc}
0 & -I_{n \times n} \\
I_{n \times n} & 0
\end{array}\right)
$$

and (2) there is a $s_{0} \in R$ such that $\exp \left(E \widetilde{B} s_{0}\right)=I$, then there is a symplectic matrix $Q$ such that $Q^{T} \widetilde{A} Q=\widetilde{A}$ and $Q^{T} \widetilde{B} Q$ has the form $\operatorname{diag}(\alpha, \beta, \gamma, \alpha, \beta, \gamma)$.

Proof. From $\widetilde{A} E \widetilde{B}=\widetilde{B} E \widetilde{A}$, we find that $\widetilde{B}$ has the following form:

$$
\widetilde{B}=\left(\begin{array}{cccccc}
a & c & 0 & 0 & e & 0 \\
c & b & 0 & e & 0 & 0 \\
0 & 0 & b_{33} & 0 & 0 & b_{36} \\
0 & e & 0 & a & -c & 0 \\
e & 0 & 0 & -c & b & 0 \\
0 & 0 & b_{63} & 0 & 0 & b_{66}
\end{array}\right)
$$

and furthermore,

$$
c=e=0 \quad \text { when } \quad k \neq 1
$$

Let

$$
B_{1}=\left(\begin{array}{cccc}
a & c & 0 & e \\
c & b & e & 0 \\
0 & e & a & -c \\
e & 0 & -c & b
\end{array}\right), B_{2}=\left(\begin{array}{cc}
b_{33} & b_{36} \\
b_{36} & b_{66}
\end{array}\right)
$$

From $\exp \left(E \widetilde{B} s_{0}\right)=I$, we can obtain $\exp \left(E B_{1} s_{0}\right)=I$ and $\exp \left(E B_{2} s_{0}\right)=I$. Now we first consider the matrix $B_{2}$. Let $\lambda$ be an eigenvalue of $E B_{2}$ with the corresponding eigenvector $\xi$, (i.e., $\left(E B_{2}\right) \xi=\lambda \xi$, ) then $\exp \left(E B_{2} s\right) \xi=e^{\lambda s} \xi$. Since $\exp \left(E B_{2} s_{0}\right)=I$, we have $e^{\lambda s_{0}}=1$. Thus $\lambda$ must be $\pm \mu i$ for $\mu \in \mathbb{R}$. If $b_{33}=0$, then $b_{36}=0$. Thus $B_{2}$ has the diagnol form and we abe done. If $b_{33} \neq 0$, without loss of generality, we can assume $b_{33}>0$. Take $w=\left(\frac{b_{33} b_{66}-b_{36}^{2}}{b_{33}^{2}}\right)^{\frac{1}{4}}$ and cosider the symplectic matrix

$$
Q_{1}=\left(\begin{array}{cc}
w & -\frac{b_{36}}{b_{33}} \frac{1}{w} \\
0 & \frac{1}{w}
\end{array}\right)
$$

then

$$
Q_{1}^{T} B_{2} Q_{1}=\left(\begin{array}{cc}
\sqrt{b_{33} b_{66}-b_{36}^{2}} & 0 \\
0 & \sqrt{b_{33} b_{66}-b_{36}^{2}}
\end{array}\right) .
$$

Next we consider the matrix $B_{1}$. From $\exp \left(E B_{1} s_{0}\right)=I$, the eigenvalues of $E B_{1}$ are $\pm \nu i$ for $\nu \in \mathbb{R}$. But by direct calculation, the eigenvalues of $E B_{1}$ are

$$
\lambda=\frac{ \pm(a-b) i \pm \sqrt{4\left(e^{2}+c^{2}\right)-(a+b)^{2}}}{2} .
$$


So we obtain

$$
4\left(e^{2}+c^{2}\right) \leq(a+b)^{2}
$$

If $a+b=0$, then $e=c=0$ and $B_{1}$ is of the diagonal form. So without loss of generality, we can assume $a+b>0$. From (4.2), we have $(a+b)^{2}-4 c^{2} \geq 0$. If $c=0$, we take $u=0$. If $c \neq 0$, we take $u=\frac{-1}{2 c}\left[(a+b)-\sqrt{(a+b)^{2}-4 c^{2}}\right]$. Notice that $1-u^{2} \geq 0$. If $1-u^{2}=0$, then $a+b= \pm 2 c$ and $e=0$. It is easy to prove that in this case there isn't any $s_{0}$ such that $\exp \left(E B_{1} s_{0}\right)=I$. Thus $1-u^{2}>0$. So we can take the symplectic matrix

$$
Q_{2}=\frac{1}{\sqrt{1-u^{2}}}\left(\begin{array}{cccc}
1 & u & 0 & 0 \\
u & 1 & 0 & 0 \\
0 & 0 & 1 & -u \\
0 & 0 & -u & 1
\end{array}\right)
$$

One easily checks that

$$
B_{3}=Q_{2}^{T} B_{1} Q_{2}=\left(\begin{array}{cccc}
a_{1} & 0 & 0 & e \\
0 & b_{1} & e & 0 \\
0 & e & a_{1} & 0 \\
e & 0 & 0 & b_{1}
\end{array}\right)
$$

where $a_{1}=\frac{1}{1-u^{2}}\left(b u^{2}+2 c u+a\right)$ and $b_{1}=\frac{1}{1-u^{2}}\left(a u^{2}+2 c u+b\right)$. One verifies

$$
\left(a_{1}+b_{1}\right)^{2}=(a+b)^{2}-4 c^{2} .
$$

Now from (4.2) and (4.3) we have $\left(a_{1}+b_{1}\right)^{2}-4 e^{2} \geq 0$. If $e=0$, we take $v=0$. If $e \neq 0$, we take $v=\frac{-1}{2 e}\left(a_{1}+b_{1}-\sqrt{\left(a_{1}+b_{1}\right)^{2}-4 e^{2}}\right)$. Again we have $1-v^{2} \geq 0$.

If $1-v^{2}=0$, then $a_{1}+b_{1}= \pm 2 e$. If we take the symplectic matrix

$$
R=\left(\begin{array}{cccc}
1 & 0 & 0 & 0 \\
0 & 0 & 0 & -1 \\
0 & 0 & 1 & 0 \\
0 & 1 & 0 & 0
\end{array}\right)
$$

then we have

$$
R^{T} B_{3} R=\left(\begin{array}{cccc}
a_{1} & e & 0 & 0 \\
e & b_{1} & 0 & 0 \\
0 & 0 & a_{1} & -e \\
0 & 0 & -e & b_{1}
\end{array}\right)
$$

As before, we can prove that $1-v^{2}=0$ is impossible.

Now we are reduced to the case $1-v^{2}>0$. We take symplectic matrix

$$
Q_{3}=\frac{1}{\sqrt{1-v^{2}}}\left(\begin{array}{cccc}
1 & 0 & 0 & v \\
0 & 1 & v & 0 \\
0 & v & 1 & 0 \\
v & 0 & 0 & 1
\end{array}\right)
$$

Then

$$
B_{4}=Q_{3}^{T} B_{3} Q_{3}=\left(\begin{array}{cccc}
a_{2} & 0 & 0 & 0 \\
0 & b_{2} & 0 & 0 \\
0 & 0 & a_{2} & 0 \\
0 & 0 & 0 & b_{2}
\end{array}\right)
$$


We let

$$
Q=\frac{1}{\sqrt{1-u^{2}}} \frac{1}{\sqrt{1-v^{2}}}\left(\begin{array}{cccccc}
1 & u & 0 & u v & v & 0 \\
u & 1 & 0 & v & u v & 0 \\
0 & 0 & w & 0 & 0 & -\frac{b_{36}}{b_{33}} \frac{1}{w} \\
-u v & v & 0 & 1 & -u & 0 \\
v & -u v & 0 & -u & 1 & 0 \\
0 & 0 & 0 & 0 & 0 & \frac{1}{w}
\end{array}\right)
$$

where $u, v$ and $w$ are taken as before. Then we can check that $Q^{T} \widetilde{B} Q$ has diagonal form. If $k=1$, we have $Q^{T} \widetilde{A} Q=\widetilde{A}$. If $k \neq 1$, from (4.1) we can take $u=v=0$. Thus $Q^{T} \widetilde{A} Q=\widetilde{A}$. This proves the Lemma.

Now we discuss the case of $(2,2,3)$.

THEOREM 18. Let $F=\left(f_{1}, f_{2}, f_{3}\right): \mathbb{C}^{3} \rightarrow \mathbb{R}^{3}$ be a homogeneous $S L$-fibration with $\left(n_{1}, n_{2}, n_{3}\right)=(2,2,3)$ so that its central fiber $L_{0}$ is a regular cone. Then there is a unitary matrix $S$ so that if we make the Darboux coordinates change

$$
\left(p_{1}, p_{2}, p_{3}, q_{1}, q_{2}, q_{3}\right)^{T}=S^{-1}\left(x_{1}, x_{2}, x_{3}, y_{1}, y_{2}, y_{3}\right)^{T}
$$

and let $w_{k}=p_{k}+i q_{k}$. Then $\left(f_{1}, f_{2}, f_{3}\right)$ is linearly equivalent to

$$
\tilde{f}_{1}=\left|w_{1}\right|^{2}-\left|w_{2}\right|^{2}, \quad \tilde{f}_{2}=\left|w_{1}\right|^{2}-\left|w_{3}\right|^{2} \quad \text { and } \quad \tilde{f}_{3}=\operatorname{Im}\left(w_{1} w_{2} w_{3}\right)
$$

Proof. Let $L_{0}=F^{-1}(0)$ and let $x_{0} \in L_{0}$. Let $\Sigma$ be a connected component of $L_{0} \cap S^{5}$ containing $x_{0}$. Let $f_{1}(x)=x^{T} A x$ and $f_{2}(x)=x^{T} B x$, where $x^{T}=$ $\left(x_{1}, x_{2}, x_{3}, y_{1}, y_{2}, y_{3}\right)$. Because $A$ and $B$ are symmetric matrices, $E A, E B \in s p(6, \mathbb{R})$, the later is the Lie algebra of symplectic group $S p(6, \mathbb{R})$. So

$$
G=\{\exp (E A t), \exp (E B s) \mid t, s \in \mathbb{R}\} \subset S p(6, \mathbb{R})
$$

is a Lie subgroup of the symplectic group. From $\left\{f_{1}, f_{2}\right\}=0$, we know that $A E B=$ $B E A$ and thus $(E A)(E B)=(E B)(E A)$. Thus $G$ is a commutative Lie subgroup of $S p(6, R)$. On $\mathbb{R}^{6}$ we define the distribution

$$
D=\left\{(E A) x,(E B) x \mid x \in \mathbb{R}^{6}\right\} .
$$

Then the distribution $D$ is completely integrable because

$[(E A) x,(E B) x]=\bar{\nabla}_{(E A) x}(E B) x-\bar{\nabla}_{(E B) x}(E A) x=(E B)(E A) x-(E A)(E B) x=0$.

Thus the orbit $G \cdot x_{0}$ is the maximal connected integral submanifold of $D$ through $x_{0}$.

On the other hand, we will prove $\Sigma$ is also the maximal connected integral submanifold of $D$ through $x_{0}$. Let $W_{3}$ be the normal deformation vector field of $L_{0}$ associated to the family $L_{s_{i}}=\left\{f_{1}=f_{2}=0, f_{3}=s_{3}\right\}$. As in the proof of Lemma 5 , we have

$$
<W_{3}, \operatorname{grad} f_{1}>=<W_{3}, \operatorname{grad} f_{2}>=0 .
$$

Because $\operatorname{deg} f_{3}=3$, from Lemma 4 , we have $\left.\theta_{3}=W_{3}\right\rfloor \omega_{0}=C_{3} t^{-2} d t$, where $C_{3}$ is a constant on $\Sigma$. Thus $W_{3}(x)=C_{3}|x|^{-3} J x$ for any $x \in \Sigma$. So from (4.4) we have equations

$$
<x,(E A) x>=<x,(E B) x>=0
$$


On the other hand $J \operatorname{grad} f_{1}=2 E A x$ and $J \operatorname{grad} f_{2}=2 E B x$ are vector fields on $L_{0}^{*}$. Then (4.5) says that $(E A) x$ and $(E B) x$ are 2 linearly independent vector fields on $\Sigma$. So $\Sigma$ is also the integral submanifold of $D$ through $x_{0}$. Thus we have $\Sigma=G \cdot x_{0}$.

On $\Sigma$, there are 2 commuting linearly independent vector fields $(E A) x$ and $(E B) x$. From [1, Lemma 2, p.274] and its proof, we know that $\Sigma$ is a 2-torus and that $\exp (E A t) \cdot x_{0}$ is a circle on $\Sigma$. Let $t_{0}$ be the first $t$ such that $\exp \left(E A t_{0}\right) x_{0}=x_{0}$. Thus for any $x=\exp (E A t) \exp (E B s) \cdot x_{0} \in \Sigma$, we have $\exp \left(E A t_{0}\right) x=x$, namely, $\left(\exp \left(E A t_{0}\right)-I\right) x=0$. But $\Sigma$ is full as the submanifold of $\mathbb{R}^{6}$ as we observed in section 1 , so we must have $\exp \left(E A t_{0}\right)=I$ and $\exp (E A t)$ is a circle on $G$. For the same reason, $\exp (E B s)$ is a circle on $G$. We let $s_{0}$ be the first $s$ such that $\exp \left(E B s_{0}\right)=I$.

Now by Williammson's theorem [9], we can reduce $A$ to normal forms by means of a real symplectic transformation. In [1, Appendix 6] we can find the list of normal forms. From $\exp \left(E A t_{0}\right)=I$, eigenvalues of $E A$ are of the form 0 or $\pm \mu i$ for $\mu \in R$. Thus we only need to discuss the case with eigenvalues 0 or $\pm \mu i$. In other words, we need to check which symmetric matrices $C$ with eigenvalues 0 or $\pm \mu i$ satisfy the equation $\exp \left(E C t_{0}\right)=I$. After that we can find a symplectic matrix $P_{1}$ such that

$$
A^{\prime}=P_{1}^{T} A P_{1}=\operatorname{diag}\left( \pm \mu_{1}^{2}, \pm \mu_{2}^{2}, \pm \mu_{3}^{2}, \pm \delta_{1}, \pm \delta_{2}, \pm \delta_{3}\right)
$$

where $\pm \mu_{j} i(j=1,2,3)$ are eigenvalues of $E A$ and $\delta_{j}=1$ if $\mu_{j} \neq 0 ; \delta_{j}=0$ if $\mu_{j}=0$. Certainly there is another symplectic matrix $P_{2}$ such that

$$
\widetilde{A}=\left(P_{1} P_{2}\right)^{T} A\left(P_{1} P_{2}\right)=\operatorname{diag}\left(r_{1}, r_{2}, r_{3}, r_{1}, r_{2}, r_{3}\right),
$$

where $r_{j}= \pm \mu_{j}(j=1,2,3)$.

Without loss of generality, we can assume that $\operatorname{det}(E A)=0$ and $\pm i$ are eigenvalues of $E A$. This is because if not, we can take $A_{1}=(u A-v B)$ for $u, v \in \mathbb{R}$ such that $\operatorname{det}\left(E A_{1}\right)=0$ and $\pm i$ are eigenvalues of $\left(E A_{1}\right)$. If we take $\bar{f}_{1}=u f_{1}-v f_{2}=u s_{1}-v s_{2}, \bar{f}_{2}=f_{2}=s_{2}$ and $\bar{f}_{3}=f_{3}=s_{3}$, then $\bar{F}=\left(\bar{f}_{1}, \bar{f}_{2}, \bar{f}_{3}\right)$ also defines homogeneous SL fibration. This SL fibration has the same geometric structure as the SL fibration $F$. This is the meaning of "linearly equivalent" in the theorem.

Let $P=P_{1} P_{2}$, then $\widetilde{A}=P^{T} A P=\operatorname{diag}(1,-k, 0,1,-k, 0)$. We note that $k>0$. Because if $k \leq 0$, then $C(\Sigma)=\{0\}$. Let $\widetilde{B}=P^{T} B P$. First we have $\exp \left(E \widetilde{B} s_{0}\right)=I$. On the other hand, from $A E B=B E A$, we have

$$
\widetilde{A} E \widetilde{B}=\left(P^{T} A P\right) E\left(P^{T} B P\right)=P^{T} A E B P=P^{T} B E A P=\widetilde{B} E \widetilde{A} .
$$

So from Lemma 17, there is a symplectic matrix $Q$ such that $Q^{T} \widetilde{A} Q=\widetilde{A}$ and $Q^{T} \widetilde{B} Q=$ $\operatorname{diag}(\alpha, \beta, \gamma, \alpha, \beta, \gamma)$.

Thus if we take $S=P Q$, then $S^{T} A S=\operatorname{diag}(1,-k, 0,1,-k, 0)$ and $S^{T} B S=$ $\operatorname{diag}(\alpha, \beta, \gamma, \alpha, \beta, \gamma)$. If we take suitable linearly transformation, we can take $S^{T} B S=$ $\operatorname{diag}(1,0,-l, 1,0,-l)$ with $l>0$.

Now if we take the Darboux coordinates

$$
\left(p_{1}, p_{2}, p_{3}, q_{1}, q_{2}, q_{3}\right)^{T}=S^{-1}\left(x_{1}, x_{2}, x_{3}, y_{1}, y_{2}, y_{3}\right)^{T}
$$

we have proven that

$$
\begin{aligned}
& \tilde{f}_{1}=\left(p_{1}^{2}+q_{1}^{2}\right)-k\left(p_{2}^{2}+q_{2}^{2}\right) \\
& \widetilde{f}_{2}=\left(p_{1}^{2}+q_{1}^{2}\right)-l\left(p_{3}^{2}+q_{3}^{2}\right) .
\end{aligned}
$$


Because Poisson bracket is preserved by the symplectic transformation, we still have $\left\{\widetilde{f}_{i}, \widetilde{f}_{j}\right\}=0$ at the Darboux coordinates $\left(p_{j}, q_{j}\right)$. From $\left\{\widetilde{f}_{j}, \widetilde{f}_{3}\right\}=0$ for $j=1,2$, we have

$$
p_{1} \frac{\partial \widetilde{f}_{3}}{\partial q_{1}}-q_{1} \frac{\partial \widetilde{f}_{3}}{\partial p_{1}}=k\left(p_{2} \frac{\partial \widetilde{f}_{3}}{\partial q_{2}}-q_{2} \frac{\partial \widetilde{f}_{3}}{\partial p_{2}}\right)=l\left(p_{3} \frac{\partial \widetilde{f}_{3}}{\partial q_{3}}-q_{3} \frac{\partial \widetilde{f}_{3}}{\partial p_{3}}\right) .
$$

Using above equations, by observation, $\widetilde{f}_{3}$ can not contain the following items:

$$
p_{i}^{2} q_{i}, p_{i} q_{i}^{2}, p_{i}^{3}, q_{i}^{3}, p_{i}^{2} p_{j}, p_{i}^{2} q_{j}, p_{i} q_{j}^{2}, q_{i} q_{j}^{2}, p_{i} p_{j} q_{i}, p_{i} q_{i} q_{j}(i \neq j) .
$$

So $\widetilde{f}_{3}$ only contains following items:

$p_{1} p_{2} p_{3}, p_{1} p_{2} q_{3}, p_{1} q_{2} p_{3}, p_{1} q_{2} q_{3}, q_{1} p_{2} p_{3}, q_{1} p_{2} q_{3}, q_{1} q_{2} p_{3}, q_{1} q_{2} q_{3}$.

Using $\left\{\tilde{f}_{i}, \tilde{f}_{j}\right\}=0$, a straight forward computation shows that $k=l=1$ and

$$
\begin{aligned}
\widetilde{f}_{3}= & a\left(p_{1} p_{2} p_{3}-p_{1} q_{2} q_{3}-q_{1} p_{2} q_{3}-q_{1} q_{2} p_{3}\right)+b\left(p_{1} p_{2} q_{3}+p_{1} q_{2} p_{3}+q_{1} p_{2} p_{3}-q_{1} q_{2} q_{3}\right) \\
& =a \operatorname{Re}\left(p_{1}+i q_{1}\right)\left(p_{2}+i q_{2}\right)\left(p_{3}+i q_{3}\right)+b \operatorname{Im}\left(p_{1}+i q_{1}\right)\left(p_{2}+i q_{2}\right)\left(p_{3}+i q_{3}\right),
\end{aligned}
$$

where $a$ and $b$ are constants. So if let $\sin \theta=\frac{a}{\sqrt{a^{2}+b^{2}}}, \cos \theta=\frac{b}{\sqrt{a^{2}+b^{2}}}$, then

$$
\widetilde{f}_{3}=\sqrt{a^{2}+b^{2}} \operatorname{I} m\left[e^{i \theta}\left(p_{1}+i q_{1}\right)\left(p_{2}+i q_{2}\right)\left(p_{3}+i q_{3}\right)\right] .
$$

So we can assume that

$$
\widetilde{f}_{3}=\operatorname{Im}\left(p_{1}+i q_{1}\right)\left(p_{2}+i q_{2}\right)\left(p_{3}+i q_{3}\right)=p_{1} p_{2} q_{3}+p_{1} q_{2} p_{3}+q_{1} p_{2} p_{3}-q_{1} q_{2} q_{3}
$$

by some unitary translation and linear translation.

Now we must prove $S \in O(6, \mathbb{R})$. Let

$$
U=\operatorname{diag}(1,-1,0,1,-1,0)
$$

and let

$$
V=\operatorname{diag}(1,0,-1,1,0,-1) .
$$

We have proven that $S^{T} A S=U$ and $S^{T} B S=V$. Then

$$
\begin{aligned}
S^{-1} \exp (E A t) S & =\exp \left(S^{-1} E A S t\right)=\exp \left(E S^{T} A S t\right) \\
& =\exp (E U t)=\operatorname{diag}\left(e^{i t}, e^{-i t}, 1\right)
\end{aligned}
$$

and

$$
S^{-1} \exp (E B s) S=\exp (E V s)=\operatorname{diag}\left(e^{i s}, 1, e^{-i s}\right) .
$$

So we have $S^{-1} G S=T^{2}=\left\{\operatorname{diag}\left(e^{i t_{1}}, e^{i t_{2}}, e^{-i\left(t_{1}+t_{2}\right)}\right) \mid t_{1}, t_{2} \in R\right\}$ or $G=S T S^{-1}$. Let $C\left(\Sigma^{\prime}\right)=\left\{f_{1}(p, q)=f_{2}(p, q)=f_{3}(p, q)=0\right\}$ and $p_{0}=\frac{1}{\sqrt{3}}(1,1,1,0,0,0)^{T} \in C\left(\Sigma^{\prime}\right)$, then there is a point $x_{0} \in \Sigma$ such that $S^{-1} x_{0}=c p_{0}$, where $c$ is a constant. From 
$G \cdot x_{0}=\Sigma \subset S^{5}(1)$, we have $\left\langle g x_{0}, g x_{0}>=1\right.$ for any $g \in G$. So for any $\tau \in T^{2}$, we have

$$
<S \tau S^{-1} x_{0}, S \tau S^{-1} x_{0}>=c^{2}<S \tau p_{0}, S \tau p_{0}>=1
$$

or

$$
\left(\tau p_{0}\right)^{T}\left(S^{T} S\right)\left(\tau p_{0}\right)=c^{-2}
$$

Let $\tau=\operatorname{diag}\left(e^{i t_{1}}, e^{i t_{2}}, e^{-i\left(t_{1}+t_{2}\right)}\right)$ and let $u=\operatorname{cost}_{1}, v=\operatorname{cost}_{2}$. Let $S^{T} S=\left(m_{i j}\right)$ and let

$$
\begin{aligned}
h(u, v)= & m_{33}+m_{44}+m_{55}-2 m_{46} v-2 m_{56} u+2 m_{12} u v \\
+ & 2\left(m_{13}+m_{46}\right) u^{2} v+2\left(m_{25}+m_{56}\right) u v^{2}+\left(m_{11}-m_{44}\right) u^{2} \\
+ & \left(m_{22}-m_{55}\right) v^{2}+\left(m_{33}-m_{66}\right)\left(2 u^{2} v^{2}-u^{2}-v^{2}\right) \\
+ & \left\{-2 m_{35}+2\left(m_{14}+m_{36}\right) u+2 m_{24} v+2\left(m_{34}-m_{16}\right) u v\right. \\
& \left.+2\left(m_{35}-m_{26}\right) v^{2}-4 m_{36} u v^{2}\right\} \sqrt{1-u^{2}} \\
+ & \left\{-2 m_{34}+2\left(m_{25}+m_{36}\right) v+2 m_{15} u+2\left(m_{34}-m_{16}\right) u^{2}\right. \\
& \left.+2\left(m_{35}-m_{26}\right) u v-4 m_{36} u^{2} v\right\} \sqrt{1-v^{2}} \\
+ & \left\{2 m_{45}-2\left(m_{13}+m_{46}\right) u-2\left(m_{23}+m_{56}\right) v\right. \\
& \left.-2\left(m_{33}-m_{66}\right) u v\right\} \sqrt{1-u^{2}} \sqrt{1-v^{2}}
\end{aligned}
$$

Then (4.6) can be rewritten

$$
h(u, v) \equiv 3 c^{-2}
$$

for any $-1 \leq u, v \leq 1$. So we have $\frac{\partial^{l} h}{\partial u^{n} \partial v^{l-n}}=0$. By direct calculation, we can get

$$
\begin{aligned}
\left.\frac{\partial^{6} h}{\partial u^{3} \partial v^{3}}\right|_{u=v=0} & =m_{33}-m_{66}=0 \\
\left.\frac{\partial^{6} h}{\partial u^{3} \partial v^{3}}\right|_{u=0} & =-18\left(m_{13}+m_{46}\right) v\left(1-v^{2}\right)^{-\frac{5}{2}}=0 \\
\left.\frac{\partial^{6} h}{\partial u^{3} \partial v^{3}}\right|_{v=0} & =-18\left(m_{23}+m_{56}\right) u\left(1-u^{2}\right)^{-\frac{5}{3}}=0 \\
\frac{\partial^{6} h}{\partial u^{3} \partial v^{3}} & =18 m_{45} u v\left(1-u^{2}\right)^{-\frac{5}{2}}\left(1-v^{2}\right)^{-\frac{5}{2}}=0
\end{aligned}
$$

From above equations, we can obtain $m_{45}=0, m_{33}=m_{66}, m_{13}=-m_{46}$ and $m_{23}=$ $-m_{56}$. Thus $h$ can be write in the following form:

$$
\begin{aligned}
h(u, v)= & m_{33}+m_{44}+m_{55}+2 m_{13} v+2 m_{23} u+2 m_{12} u v \\
+ & \left(m_{11}-m_{44}\right) u^{2}+\left(m_{22}-m_{55}\right) v^{2} \\
+ & \left\{-2 m_{35}+2\left(m_{14}+m_{36}\right) u+2 m_{24} v+2\left(m_{34}-m_{16}\right) u v\right. \\
& \left.+2\left(m_{35}-m_{26}\right) v^{2}-4 m_{36} u v^{2}\right\} \sqrt{1-u^{2}} \\
+ & \left\{-2 m_{34}+2\left(m_{25}+m_{36}\right) v+2 m_{15} u+2\left(m_{34}-m_{16}\right) u^{2}\right. \\
& \left.+2\left(m_{35}-m_{26}\right) u v-4 m_{36} u^{2} v\right\} \sqrt{1-v^{2}}
\end{aligned}
$$


Using the same method, at last, we can obtain

$$
S^{T} S=\operatorname{diag}\left(m_{11}, m_{22}, m_{33}, m_{11}, m_{22}, m_{33}\right)
$$

and

$$
m_{11}+m_{22}+m_{33}=3 c^{-2} .
$$

But $S$ is the symplectic matrix, so $S^{-1}$ and $S^{T}$ are symplectic matrices too. Thus we have

$$
S^{T} S E S^{T} S=S^{T} E S=E
$$

Now from (4.8) and (4.9), we easily can get $m_{11}=m_{22}=m_{33}=1$. From above discussion, we have $S^{T} S=I$ and $S \in O(6, \mathbb{R})$. Thus we have proven $S \in U(3)$ and this completes the proof of theorem 18.

\section{REFERENCES}

[1] V.I. ARnold, Mathematical Methods of Classical Mechanics, Spinger-Verlag, Second Edition.

[2] A.A. GRIGOR'YAN, Acriterion for the existence on a Riemannian manifold of a nontrival bounded harmonic function with finite Dirichlet integral, Soviet Math Dolk, 35 (1987), pp. 339-341.

[3] F.R. Harvey and H.B. Lawson, Calibrated geometries, Acta Math., 148 (1982), pp. 47-157.

[4] N. Hitchin, $L^{2}$-cohomology of hyperkaehler quotients, math.DG/9909002.

[5] D. JoYCE, On counting special Lagrangian homology 3-spheres, V1, hep-th/9907013.

[6] P. LI AND L.F. TAM, Green's functions, harmonic functions, and volume comparisson, J.Diff.Geom., 41 (1995), pp. 277-318.

[7] J. Simons, Minimal varieties in riemannian manifoldds, Ann.Math., 88 (1968), pp. 62-105.

[8] A. Strominger, S.-T. Yau And E. Zaslow, Mirror Symmetry is T-duality, Nucl. Phys.B., 479 (1996), pp. 243-259.

[9] J. Williamson, On an algebraic problem concerning the normal forms of linear dynamical systems, Amer. J. of Math., 58 (1936), pp. 141-163.

[10] Z-F. Zhang, T-R. Ding, W-Z. Huang And Z-X. Dong, Stability Theory of differentiable equations, Scientific Press, China. 